\title{
Vicisitudes de la labor docente en un contexto de violencia por el crimen organizado
}

Vicissitudes of teaching because of a criminal gang's context of violence

Vicissitudes do ensino em contexto de violência do crime organizado

Santos Noé Herrera Mijangos

Universidad Autónoma del Estado de Hidalgo, México

psicologonoe@yahoo.com

https://orcid.org/0000-0001-6567-0986

Dayana Luna Reyes

Universidad Autónoma del Estado de Hidalgo, México dayis2902@gmail.com https://orcid.org/0000-0001-9687-2521

Jorge Gonzalo Escobar Torres

Universidad Autónoma del Estado de Hidalgo, México jorgegonzaloescobar@gmail.com http://orcid.org/0000-0002-9598-8781

Janet Serrano Díaz

Universidad Autónoma del Estado de México, México janet_sd26@hotmail.com http://orcid.org/0000-0001-7206-4132 


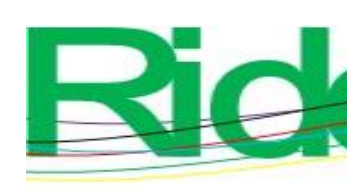

Revista Iberoamericana para la Investigación y el Desarrollo Educativo ISSN $2007-7467$

\section{Resumen}

La institución educativa tiene el mandato social de transmitir la cultura de la comunidad que la sostiene, pero en muchas ocasiones olvidan su función básica porque padecen de violencias, marginación y pobreza, entre otras problemáticas. La metodología de esta investigación es cualitativa, de corte descriptivo e interpretativo. El objetivo consistió en describir las diversas violencias externas e internas que sufren los profesores de un Colegio de Bachillerato Tecnológico ubicado en el Estado de México. Se realizaron entrevistas en profundidad y grupos focales, así como observaciones naturalistas sustentadas en el análisis institucional, también conocido como etnografía escolar. Los resultados evidencian violencia externa perpetrada por el crimen organizado, así como violencia interna ejercida por el director y los estudiantes. Ambas cuestiones generan que los profesores cedan en su labor docente por las amenazas de algunos delincuentes y ante la incompetencia del Estado, la laxitud de las normas institucionales, la falta de apoyo de la sociedad, los directivos y los padres de familia. Las violencias en la institución de análisis son un reflejo de la dinámica social, económica y política, similar a los resultados encontrados en otras investigaciones del país. La escuela tiene episodios alarmantes de violencia, al igual que muchas otras alrededor del mundo, y ha alcanzado un estatuto de naturalización como fenómeno social. De igual manera, se advierte cómo la educación formal pierde importancia en términos de movilidad social para los estudiantes debido a las ganancias económicas que obtienen de forma inmediata por participar con el crimen organizado.

Palabras clave: coeducación, crimen organizado, escuelas, violencia.

\section{Abstract}

The educational institution has a social mandate. It has to transmit the culture of the community, but sometimes it is forgotten because it suffers from violence, marginalization and poverty, among other problems. The methodology of this research is qualitative, descriptive and interpretive. The objective of this research was to describe the external and internal violence suffered by teachers in a Technological Bachelorship College located in the State of Mexico. Some interviews and naturalistic observations were done. The results show external violence perpetrated by criminal gangs, as well as internal violence carried out by the principal and students. Both issues affect teacher's work and they accept threats of some criminals and the incompetence of the State, the laxity of institutional norms, the 


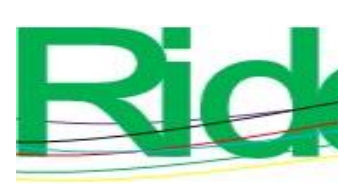

Revista Iberoamericana para la
Investigación y el Desarrollo Educativo
ISSN $2007-7467$

lack of support from society, managers and parents. Violence in the institution of analysis is a reflection of the social, economic and political dynamics, similar to the results found in other investigations in the country. The school has alarming episodes of violence, like many others around the world. It has achieved naturalization status as a social phenomenon. In the same way, it is noted that formal education loses importance for students because of economic gains. They obtain easy money participating with criminal gangs.

Keywords: Coeducation, criminal gangs, schools, violence.

\section{Resumo}

A instituição educacional tem o mandato social de transmitir a cultura da comunidade que a apóia, mas em muitas ocasiões se esquece de sua função básica porque sofre com a violência, a marginalização e a pobreza, entre outros problemas. A metodologia desta pesquisa é qualitativa, descritiva e interpretativa. $\mathrm{O}$ objetivo foi descrever as diversas violências externas e internas sofridas pelos professores de uma Escola Superior Tecnológica localizada no Estado do México. Foram realizadas entrevistas em profundidade e grupos focais, bem como observações naturalísticas apoiadas em análises institucionais, também conhecidas como etnografia escolar. Os resultados mostram a violência externa perpetrada pelo crime organizado, bem como a violência interna perpetrada pela diretora e pelos alunos. Ambas as questões fazem com que os professores desistam do trabalho docente devido às ameaças de alguns criminosos e à incompetência do Estado, à negligência das normas institucionais, à falta de apoio da sociedade, gestores e pais. A violência na instituição de análise é um reflexo da dinâmica social, econômica e política, semelhante aos resultados encontrados em outras investigações no país. A escola vive episódios alarmantes de violência, como tantas outras no mundo, e alcançou o status de naturalização como fenômeno social. Da mesma forma, nota-se como a educação formal perde importância em termos de mobilidade social dos alunos devido aos ganhos econômicos que obtêm imediatamente com a participação no crime organizado.

Palavras-chave: coeducação, crime organizado, escolas, violência.

Fecha Recepción: Junio 2020

Fecha Aceptación: Enero 2021 


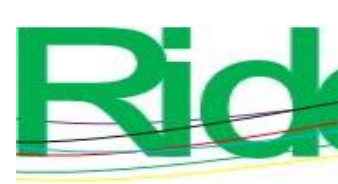

Revista Iberoamericana para la Investigación y el Desarrollo Educativo ISSN 2007-7467

\section{Introducción}

Las instituciones "son una creación de la sociedad para organizar, proteger y dar seguridad a los seres humanos" (Herrera, Luna y Barojas, 2019a, p. 80). Según Manero (1990), "hablar de institución nos remite a establecimientos específicos, con su base material, su existencia visible: una asociación, una universidad" (p. 152). Aunque existen diversos tipos de instituciones, el presente artículo se enfoca en la educativa porque tiene el mandato social de "asegurar la continuidad de la comunidad que la sostiene a través de la transmisión de la cultura" (Fernández, 1996, p. 108). Dicha herencia cultural se transmite por medio de sus miembros más capaces "por poseer un alto grado de espíritu de patriotismo, fidelidad, obediencia, coraje y simpatía, siempre dispuestos a ayudarse unos a otros, y a sacrificarse por el bien común" (Darwin, 1874, citado por Lewis y Steinmo 2011, p. 125). Coincidiendo con Darwin (1874) y Fernández (1996), tanto la comunidad como la institución educativa tienen la tarea primaria de asegurar la continuidad de la cultura por medio de la fidelidad, obediencia, coraje, simpatía y bien común, aunque en muchas ocasiones olvidan su función básica porque son influenciadas por el contexto externo e interno que padecen: violencia, delincuencia, marginación, pobreza, entre otras.

En cuanto a la pobreza, caldo de cultivo de muchas problemáticas, el consejero presidente del Instituto Nacional para la Evaluación de la Educación (INEE) reconoció que "la pobreza educativa de una escuela acentúa las deficiencias de los alumnos y, en consecuencia, propicia bajos niveles de aprendizaje (...). Por desgracia, la mayoría de los estudiantes pobres de México asisten a escuelas con carencias graves (Gil, 2018, p. 307). Sumado a la pobreza, las escuelas son anarquías organizadas (Buendía, 2011), campos de lucha divididas por conflictos (Ball, 1994) internos entre sus actores institucionales. De acuerdo con Buendía (2011), son caracterizadas por "la falta de coordinación entre individuos y colectividades; la ausencia relativa de regulaciones" (p. 10).

Las instituciones educativas, al ser una caja de resonancia de la sociedad, deben ser analizadas como pequeñas sociedades desde la etnografía escolar, tratando de entender “cómo vive la gente y qué la motiva; sus relaciones con los demás, las reglas (en gran parte implícitas) que dirigen su comportamiento, el significado de las formas simbólicas tales como el lenguaje, la apariencia, y la conducta” (Vázquez y Valenzuela, 2013, p. 13). Asimismo, se debe indagar la violencia y el sufrimiento que padecen los actores institucionales que muchas veces dejan de lado la tarea primaria y evitan que los estudiantes logren determinados 


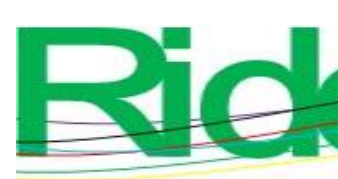

Revista Iberoamericana para la
Investigación y el Desarrollo Educativo
ISSN $2007-7467$

aprendizajes que los hagan aptos para vivir en su contexto social.

Diversas investigaciones en el mundo han abordado la problemática de la violencia externa e interna de las instituciones educativas (Remedi, 2004; Reyes-Argona, Gudiño y Fernández, 2018; Velázquez, 2011). No importa el país, religión o estatus económico, la violencia está presente en la mayoría de las instituciones educativas. Según Velázquez (2011), es pertinente "diferenciar entre violencia contra la escuela y violencia dentro de la escuela (...), [así como] de afuera hacia dentro y de dentro hacia fuera” (p. 17).

Para nuestros propósitos, en este artículo se analiza la violencia de afuera hacia adentro y dentro de la escuela. El objetivo central es describir el contexto externo e interno de un colegio de bachillerato tecnológico (CBT) ubicado en el Estado de México, México. Se busca responder qué tipo de violencias padecen los profesores en el CBT y quiénes son los actores que ejercen violencia sobre los docentes.

La palabra violencia proviene del latín vis ('fuerza'). Según Blair (2009), no hay una definición conceptual como tal, solo aproximaciones a lo que implica el fenómeno de análisis. A quien hable de violencia, siempre hay que preguntarle a qué tipo se refiere. Por tanto, la violencia que se ejerce en las instituciones educativas la definimos como el maltrato físico, psíquico y social dentro y fuera de la institución educativa. Esta violencia puede ser presencial, virtual o simbólica hacia a un estudiante, profesor y directivo, entre otros. Puede presentarse de manera única o sistemática, y genera sufrimiento latente o manifiesto en la víctima de forma temporal o permanente llegando inclusive hasta la muerte.

La violencia en las escuelas ha sido minimizada y es tan frecuente que ha sido naturalizada la problemática por el Estado y por la sociedad en diversas partes del mundo. Alemania, Canadá, Reino Unido y Escocia (Masse, 18 de enero de 2017), entre otros países, han sufrido sucesos siniestros, aunque Estados Unidos presenta los mayores índices. Por ejemplo, en el periodo escolar 2011-2012, “el $10 \%$ de los maestros de escuelas públicas K12 (Kindergarden-Twelve), por sus siglas en inglés, informó haber sido amenazado por estudiantes en los últimos 12 meses, y el $6 \%$ reportó haber sido atacado físicamente" (Huang, Eddy y Camp, 2017, citados por Abril-Martínez, 2020, p. 197). Los eventos más violentos y trágicos por el número de víctimas en Estados Unidos fueron el tiroteo en la Universidad de Texas, Austin, el 1. ${ }^{\circ}$ de agosto de 1966; la masacre en Columbine High School, Colorado, el 20 de abril de 1999; el tiroteo en Virgina Polytechnic Institute, Virginia, el 16 de abril de 2007; y la masacre en Sandy Hook Elementary School, Connecticut, el 14 de diciembre de 2012. 

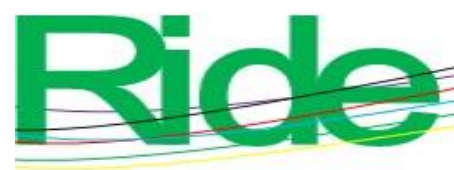

Revista Iberoamericana para la Investigación y el Desarrollo Educativo ISSN 2007 - 7467

En el caso de México, se identifican la masacre del Colegio Americano del Noreste, en Monterrey, Nuevo León, el 18 de enero de 2017, donde un menor disparó contra tres de sus compañeros y su maestra (Milenio, 18 de enero de 2017); y la masacre del Colegio Cervantes, en Torreón, Coahuila, el 10 de enero de 2020, donde un adolescente de 11 años disparó contra su maestra y compañeros (Aristegui Noticias, 13 de enero de 2020). En ambos colegios privados, las profesoras perdieron la vida.

En el terreno nacional, algunas investigaciones (Cano y Estrada, 2015; Romero, Loza y Machorro, 2013) afirman que la violencia ejercida por los estudiantes hacia sus compañeros y profesores son reflejo de la dinámica social, económica y política, lo que impacta en la escuela y en los distintos actores sociales, principalmente en los profesores, por lo que se coincide con el siguiente planteamiento de Abril-Martínez (2020):

[El profesor] no encuentra apoyos a nivel institucional, ni en las relaciones de reconocimiento con sus pares, ni en los padres de familia, propiciando sentimientos de soledad frente a la incertidumbre de la labor educativa y al fracaso de estrategias pedagógicas (p. 199).

Como se aprecia, algunos profesores han perdido no solo el respeto de la sociedad y de sus instituciones, sino incluso la vida (Herrera, Luna y Barojas, 2019a). Lo presentado previamente demuestra que la violencia ejercida contra los profesores es un reflejo de lo que se vive en las naciones y "como fenómeno social, alcanza un estatuto de naturalización" (Sáez, 2017, p. 568). Ha sido una consecuencia de la descomposición social y falta de apoyo del Estado y la sociedad en su conjunto, de ahí la importancia de los resultados de esta investigación.

\section{Método}

Esta investigación es cualitativa, de corte descriptivo e interpretativo. Se sustenta en el análisis institucional, también conocido como etnografía escolar. De acuerdo con Vázquez y Valenzuela (2013), "la etnografía de la escuela puede tener un interés inmediatamente práctico para los profesores. Se ocupa de sus problemas como individuos y no solo como miembros de una profesión" (p. 13). 


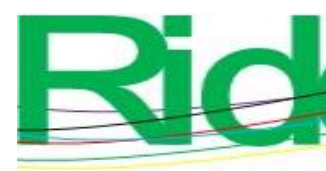

Revista Iberoamericana para la Investigación y el Desarrollo Educativo ISSN $2007-7467$

\section{Participantes}

Participaron tres profesores: dos mujeres y un hombre. Su antigüedad como educadores oscila entre los dos y los siete años. Los tres son originarios de la comunidad. De igual manera, participaron siete orientadores, algunos originarios de la comunidad y otros provenientes de poblaciones cercanas. La muestra elegida para el estudio es no probabilística y se tomó de acuerdo con el criterio de conveniencia (Hudelson, 1994). En cuanto a los criterios de inclusión, se consideró a profesores y orientadores que laboran en el CBT de análisis y aceptaron voluntariamente participar en la investigación. El criterio de exclusión fue para aquellos profesores y orientadores que no laboran en el CBT de análisis y el criterio de eliminación fue para aquellos profesores y orientadores que han dejado de trabajar en la institución.

\section{Escenario}

La investigación se realizó en un CBT ubicado en la zona norte del Estado de México. La institución educativa se encuentra cerca de la autopista México-Tuxpan, en una zona con serias carencias de infraestructura. No hay industria, hospitales ni atractivos turísticos. La población es de nivel económico bajo.

\section{Consideraciones éticas}

Los informantes decidieron colaborar voluntariamente, y para protección de los implicados los fragmentos de entrevista que se presentan son anónimos. Se utilizaron las siguientes claves para las entrevistas individuales: profesora 1 (PF1), profesor 2 (PM2), profesora 3 (PF3). En las entrevistas grupales las claves fueron orientador 1 (GO1), orientador $2(\mathrm{GO} 2)$, orientador $3(\mathrm{GO} 3)$, orientador $4(\mathrm{GO} 4)$, orientador 5 (GO5), orientador 6 (GO6) y orientador 7 (GO7). Cabe destacar que se tiene grabada la autorización de los entrevistados para publicar los resultados con fines académicos. 


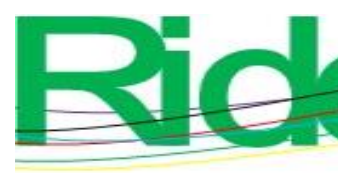

Revista Iberoamericana para la Investigación y el Desarrollo Educativo ISSN $2007-7467$

observaciones, aunque con registros fotográficos que ayudan a conservar lo que los investigadores pueden olvidar.

Una vez obtenidos los ejes y categorías de las entrevistas, y luego de analizar los formatos de observación y las fotografías, se realizó la novela institucional que consistió en tres partes: 1) creación de la narración con la información proporcionada en las entrevistas, 2) análisis de las observaciones naturalista y notas de campo y 3) análisis de fotografías tomadas en los espacios e intersticios de los actores institucionales. Estas tres partes se entrelazaron creando una sola narración, una sola novela. Vale indicar que durante la redacción se sostuvieron pláticas con el contacto que permitió el acceso a la investigación, lo que sirvió para esclarecer dudas sobre la institución y los participantes.

\section{Resultados}

\section{Violencia externa: entre la delincuencia moderada y severa}

Los CBT son instituciones educativas que viven inmersas en un constante contexto de violencia externa e interna. En cuanto a la primera, no importa el rol de los actores institucionales (estudiantes, profesores, directivos y administrativos, entre otros), pues la mayoría son víctimas de la delincuencia, ya que son alcanzados por cualquier tentáculo del crimen. Si bien es cierto que existe delincuencia moderada (robo de autos, extorciones telefónicas, amenazas de muerte), también es cierto que la delincuencia severa (secuestro, decapitados, robo de hidrocarburos — huachicol — y distribución de drogas) despierta fobias que paralizan a los actores institucionales porque los delitos son cometidos por el crimen organizado.

Durante la observación naturalista, se platicó con algunos docentes que definieron los términos de la delincuencia moderada y severa en cuanto a delitos moderados. Al respecto, una profesora comenta: "Se han robado coches de compañeros afuera de la escuela. A una cuadra tenemos familias completas que se dedican a robar, sabemos quiénes son. Hace unos días se escucharon ráfagas de disparos" (PF1). Durante la observación se ratificó que la puerta principal del CBT y parte de las paredes tienen orificios de balas. En cuanto a delitos severos, los orientadores mencionan: 


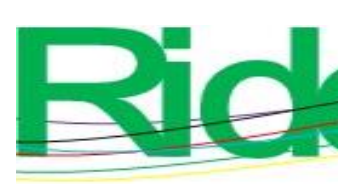

Revista Iberoamericana para la

Investigación y el Desarrollo Educativo

ISSN $2007-7467$

\section{Discusión}

La investigación alcanzó el objetivo de describir el contexto externo e interno del CBT. De igual manera, los hallazgos respondieron a las preguntas de investigación sobre las diversas violencias que sufren los profesores y focalizó a los actores institucionales que las ejercen. Los hallazgos están relacionados con otros estudios (Herrera, Grijalva y Luna, 2017) que afirman que el crimen organizado está instalado en la sociedad e instituciones y forma parte de la cotidianidad de las personas. La figura 1 resume las diferentes violencias y los actores que las ejercen cotidianamente en el CBT.

Figura 1. Tipos de violencia que padecen los profesores

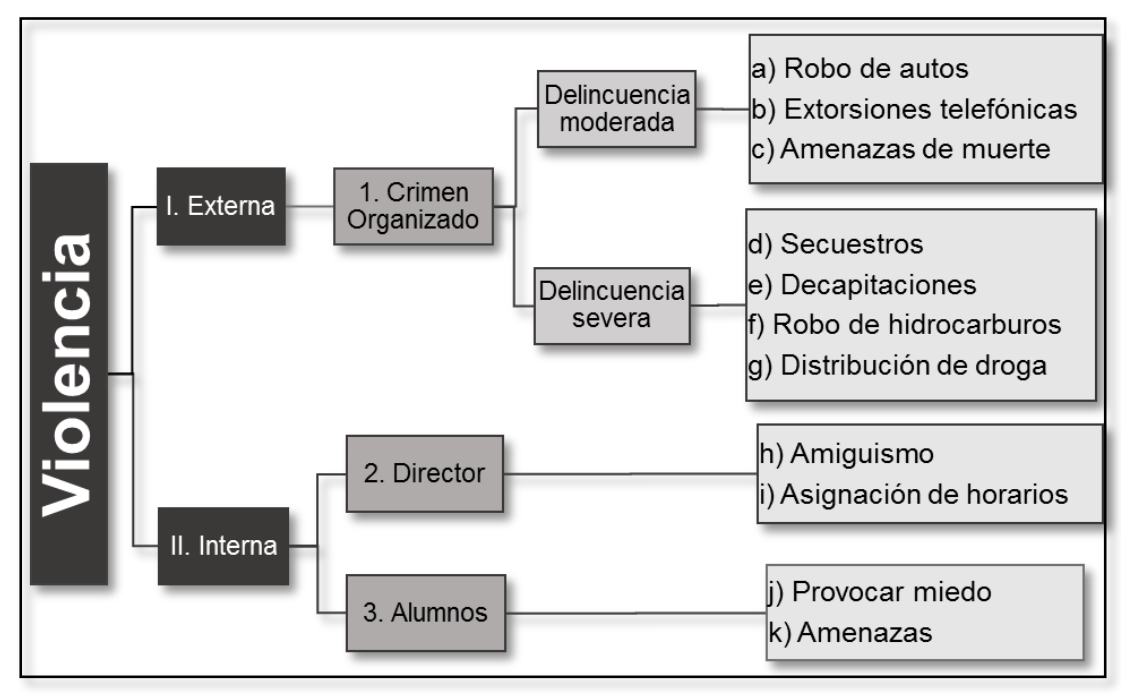

Fuente: Elaboración propia

La figura 1 devela que existe violencia externa perpetrada por el crimen organizado, así como violencia interna ejercida por el director y los alumnos. Los resultados de esta investigación tienen relación y similitud con lo referido por Velázquez (2011) cuando afirma que hay violencia "dentro de la escuela (...), [así como] de afuera hacia dentro" (p. 17). La violencia externa se explica por la pobreza, descomposición social y porque algunas familias de los estudiantes apoyan actos delictivos como el robo de autos, extorsiones telefónicas, secuestros, huachicol y distribución de drogas, entre otras actividades ilícitas. Estos sujetos anteponen sus intereses personales al bien común, con lo cual condenan a la institución, a su pueblo y a la sociedad porque "una sociedad formada únicamente por individualistas egoístas no podría durar mucho tiempo. [Aunque] por fortuna, las sociedades humanas reales están compuestas por individuos que poseen diversas preferencias y motivaciones, desde el 


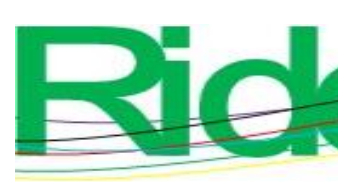

\section{Revista Iberoamericana para la Investigación y el Desarrollo Educativo ISSN $2007-7467$}

egoísmo extremo hasta el altruismo inspirador" (Lewis y Steinmo, 2011, p. 131).

En nuestra institución de análisis (incrustada en una comunidad de nivel económico bajo, con serias carencias de infraestructura), existen (pese a las circunstancias que sufren) profesores comprometidos con la labor de "asegurar la continuidad de la comunidad que la sostienen a través de la transmisión de la cultura que la define como tal" (Fernández, 1996, p. 108), pero también células del crimen organizado que influencian y cooptan a los estudiantes; en pocas palabras, los profesores procuran cumplir con su labor formativa, aunque con la constante amenaza y miedo.

En cuanto a la violencia interna (Blair, 2009) contra los profesores, los hallazgos focalizaron que el director y los estudiantes son los principales hacedores, los cuales provocan condiciones deplorables en la dinámica institucional. El director asigna horas por amiguismo y quienes no entran al juego son amenazados, condicionados y expulsados de la institución. La perversa asignación de horarios y carga de materias lastima la economía de los docentes porque dependen de ello para completar un salario digno. Cuando tienen una mala asignación, se deben desplazar a diferentes escuelas para impartir clases. Muchos profesores en México se han convertido en chambistas, aboneros y recientemente bautizados "Uber profes”. De acuerdo con Buendía (2011), las dinámicas institucionales en el CBT son una anarquía organizada.

Asimismo, se detectó que la violencia que ejercen los estudiantes hacia los profesores en el CBT son un reflejo de la dinámica social, económica y política, similar a los resultados relacionados con otras investigaciones del país (Cano y Estrada, 2015; Romero et al., 2013). Prácticamente en todos los sistemas educativos existe este problema (Herrera, Luna y Barojas, 2019a; Herrera, Luna y Escobar, 2019b), naturalizado y "asociado a la pérdida de legitimidad y de la autoridad educativa y a la escisión del acuerdo entre familias e instituciones educativas" (Eljach, 2011, citado por Abril-Martínez, 2020, p. 196).

El Estado, la sociedad y los padres de familia han dejado la mayoría de la responsabilidad al docente sin ofrecer las condiciones adecuadas. Los profesores deben contener la violencia que es importada de la comunidad y hogares a las escuelas. Por eso, coincidimos con Salinas et al. (2002) cuando afirman que el maestro está presionado por "los altos niveles de violencia e impunidad, que tocan a diario las escuelas, y reclaman de su labor resultados tangibles" (p. 247). Los resultados del CBT coinciden con Abril-Martínez (2020) cuando explica que los profesores carecen de apoyos a nivel institucional y por parte de los padres de familia, "propiciando sentimientos de soledad frente a la incertidumbre de la labor 


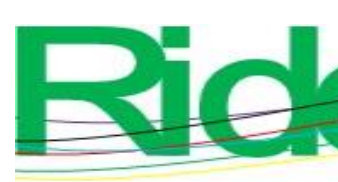

Revista Iberoamericana para la
Investigación y el Desarrollo Educativo
ISSN $2007-7467$

educativa" (p. 199).

El Estado ignora las problemática de la violencia en las escuelas, y solo las trata cuando hay masacres que implican vidas humanas y cuando se emiten informes de trabajo. Para las instituciones educativas lo más importante es rendir cuentas satisfactorias ante los organismos reguladores y muchos padres de familia dejan el problema a los profesores, pues lo importante es que su hijo apruebe para el siguiente ciclo escolar. En el CBT y en muchas partes del mundo, la violencia tiene episodios alarmantes. Esto, según Sáez (2017), ha alcanzado un estatuto de naturalización.

De igual manera, concordamos en que se han cambiado las relaciones entre los profesores y los estudiantes, "y esto hace que, al ver al docente como un par, se facilite que el estudiante apele a la agresión física o verbal como forma de dirimir los conflictos generados en la convivencia cotidiana" (Abril-Martínez, 2020, p. 198). La violencia que sufren los profesores por parte de sus estudiantes, en muchas ocasiones, es velada por miedo a perder la credibilidad ante los directivos y sus pares, de ahí la negación y ocultamiento de una realidad insostenible. En la institución de análisis, existen dos principales tipos de amenaza (secuestro y extorsión telefónica) que algunos estudiantes hacen a los profesores por no tener una calificación aprobatoria. Cabe destacar que sus coacciones se sustentan por tener vínculos con el crimen organizado, y ante eso los profesores ceden la calificación. En muchas escuelas del nivel medio superior, así como secundarias los estudiantes son "promovidos independientemente de sus logros académicos" (Weiss, 2015, citado por Saccone, 2016, p. 131). Según Saccone (2016), en una entrevista que realizó a una profesora, "si tienes una alta tasa de reprobación no entras a estímulos [gratificación económica] (...). Los jóvenes entrevistados afirman que algunos docentes prácticamente ‘te regalan’ puntos” (p. 134).

Finalmente, en cuanto a los resultados de esta investigación, es prudente mencionar que existen limitantes, implicaciones y sugerencias que deben ser consideradas. La investigación es un caso único, por lo que no se puede hacer una representación social. Aunque existen violencias y actores institucionales similares en muchas escuelas, la institución de análisis tiene características únicas por el lugar en que se encuentra, así como su único contexto social, económico y cultural, entre otras particularidades.

Una segunda limitante es la muestra tan reducida. En este sentido, vale acotar que aunque la invitación se realizó a todos los profesores, pocos aceptaron, tal vez por medio a ser castigados por los directivos o violentados por el crimen organizado. Aun así, aquellos que accedieron a participar en la investigación permitieron realizar libremente las entrevistas, 


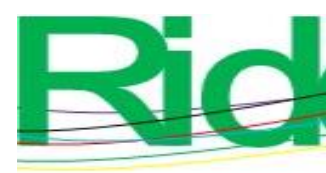

Revista Iberoamericana para la
Investigación y el Desarrollo Educativo
ISSN $2007-7467$

observaciones naturalistas y la antropología visual.

La implicación de los investigadores también pudo ser una variable que influyó en la selección de los resultados, ya que, de alguna u otra manera, formamos parte del entramado de las instituciones educativas. Aunque se trató de ser lo más objetivo posible, siempre existe en la investigación cualitativa la selección, orden e interpretación de los resultados desde la visión del investigador. Por eso, considerando estas limitantes e implicaciones, se sugiere continuar con la línea de investigación en otras escuelas del país.

\section{Conclusiones}

Las diferentes manifestaciones de violencia que padece la sociedad alrededor del mundo se han naturalizado, y las instituciones educativas al ser una caja de resonancia de la sociedad son productoras y reproductoras de esa realidad. Las violencias que sufren los actores institucionales en las escuelas evidencian que el Estado, la sociedad, las autoridades educativas y los padres de familia han perdido la batalla. Los profesores libran luchas cotidianas con actores externos, como delincuentes (a veces ocultos) y actores internos que convergen cotidianamente. El crimen organizado está plantado como una enredadera en el jardín, y forma parte de la cotidianidad de las instituciones. Lamentablemente, la institución de análisis (y muchas otras en México) se encuentran en un área geográfica fértil para el crimen organizado por la pobreza, la descomposición social y el consentimiento de algunos miembros de la sociedad que apoyan actos ilícitos.

Finalmente, es prudente reconocer la labor de los profesores porque coeducan con el crimen organizado ante el abandono de la sociedad, incompetencia del Estado, laxitud de normas institucionales, falta de apoyo de la sociedad, padres de familia y directivos. Los profesores y delincuentes compiten por la labor formativa de los alumnos y, en ocasiones, no hay parámetros académicos por las amenazas y el miedo. En este contexto, algunos estudiantes han preferido delinquir en la noche que estudiar en la mañana. 


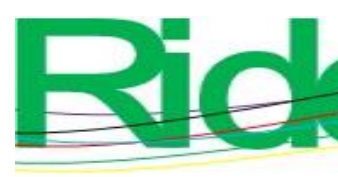

Revista Iberoamericana para la
Investigación y el Desarrollo Educativo
ISSN $2007-7467$

Futuras líneas de investigación

Durante y al final de la investigación se encontraron tres temas que llamaron la atención: 1. El modelo educativo por la eliminación de la reforma educativa, 2. Perspectiva sobre el contexto de la institución desde la visión de los padres de familia y, 3. La disonancia que enfrentan los alumnos ante la seducción del crimen organizado. Cabe destacar que los temas encontrados escaparon a los objetivos planificados en esta investigación, pero son vetas que serán desarrolladas próximamente.

\section{Referencias}

Abril-Martínez, C. A. (2020). Malestar docente y violencia escolar una relación por definir: revisión documental de la década del 90 a la actualidad. Revista Logos, Ciencia y Tecnología, 12(1), 188-205. Recuperado de http://dx.doi.org/10.22335/rlct.v12i1.1045

Aristegui Noticias (13 de enero de 2020). Armas usadas por niño en colegio de Torreón eran de su abuelo: Fiscalía de Coahuila. Aristegui Noticias. Recuperado de https://aristeguinoticias.com/1301/mexico/armas-usadas-por-nino-en-colegio-detorreon-eran-de-su-abuelo-fiscalia-de-coahuila/

Ball, S. (1994). La micropolítica de la escuela. Hacia una teoría de la organización escolar. Barcelona: Paidós.

Blair, E. (2009). Aproximación teórica al concepto de violencia: avatares de una definición. Revista Política y Cultura, (32), 9-33. Recuperado de http://www.scielo.org.mx/pdf/polcul/n32/n32a2.pdf

Buendía, M. A. (2011). Análisis institucional y educación superior. Aportes teóricos y resultados empíricos. Perfiles Educativos, 33(134), 8-33. Recuperado de https://doi.org/10.22201/iisue.24486167e.2011.134.27938

Cano, A. y Estrada, M. J. (2015). Violencia estructural y estudiantes de escuelas secundarias del noroeste fronterizo de Chihuahua, México. Eleuthera, 12, 34-55.

Fernández, L. (1996). Instituciones educativas. Dinámicas institucionales en situaciones críticas. Argentina: Paidós.

Gil, M. (2018). La reforma educativa. Fracturas estructurales. Revista Mexicana de Investigación Educativa, 23(76), 303-321. Recuperado de http://www.scielo.org.mx/scielo.php?script=sci_arttext\&pid=S1405- 


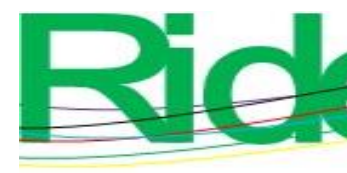

66662018000100303

Herrera, S. N., Grijalva, O. y Luna, D. (2017). ¡Ahí viene el lobo! Sufrimiento del ser y el Estado ante el crimen organizado. En Vallejo, R. y Ortega, M. C. (coords.), Distintos abordajes clínicos de las manifestaciones de la violencia (pp. 45-57). México: Fontamara.

Herrera, S. N., Luna, D. y Barojas, J. (2019a). Análisis instituciones de primarias: reforma educativa como detonante del sufrimiento docente. Revista Somepso, 3(2), 74-97. Recuperado de https://somepso.files.wordpress.com/2019/03/v3n2_4-anc3a1lisisinstitucional-de-primarias_noc3a9-herrera-1.pdf

Herrera, S. N., Luna, D. y Escobar, J. G. (2019b). Síndrome adquirido por el trabajo académico en instituciones educativas latinoamericanas. Archivos Analíticos de Políticas Educativas, 27(91). Recuperado de https://epaa.asu.edu/ojs/article/download/3763/2284

Hudelson, P. (1994). Qualitative Research for Health Programmes. Geneva: World Health Organization.

Lewis, O. y Steinmo, S. (2011). Tomemos en serio la evolución: análisis institucional y teoría evolutiva. Revista de Economía Institucional, 13(24), 111-151. Recuperado de https://revistas.uexternado.edu.co/index.php/ecoins/article/view/2843

Manero, R. (1990). Introducción al análisis institucional. Tramas, 1, 121-157. Recuperado de https://www.srmcursos.com/pdf/biblio_psicologia/manero_brito.pdf

Masse, F. (18 de enero de 2017). Top 7: Los peores tiroteos y masacres en escuelas. Milenio. Recuperado de https://www.milenio.com/cultura/top-7-peores-tiroteosmasacres-escuelas

Milenio (18 de enero de 2017). Murió autor de tiroteo en colegio de Monterrey. Milenio. Recuperado de https://www.milenio.com/politica/murio-autor-de-tiroteo-encolegio-de-monterrey

Remedi, E. (2004). Instituciones educativas. Sujetos, historia e identidades. México: Plaza y Valdés.

Reyes-Argona, S., Gudiño, S. y Fernández, J. M. (2018). Violencia escolar en Michoacán y Nuevo León, un diagnóstico situado para promover escuelas seguras en educación básica. Revista Electrónica de Investigación Educativa, 20(2), 46-58. Recuperado de https://doi.org/10.24320/redie.2018.20.2.1548

Romero, M. V., Loza, J. L. y Machorro, F. (2013). Violencia del crimen organizado 


\section{Revista Iberoamericana para la Investigación y el Desarrollo Educativo ISSN 2007 - 7467}

relacionada a los sectores económicos en México. Una propuesta de categorización. Polis Revista Latinoamericana, 36, 1-14. Recuperado de https://journals.openedition.org/polis/9624

Saccone, M. (2016). La obligatoriedad de la educación media superior. Apuntes para pensar la experiencia mexicana. Anuario de la Facultad de Ciencias Humanas UNLPam, 13(13), 122-139. Recuperado de http://dx.doi.org/10.19137/an1309

Sáez, V. (2017). Miradas sobre la escuela secundaria en situaciones de violencia. Un análisis desde la prensa argentina. Revista Mexicana de Investigación Educativa, $22(73)$, $565-583$.

Recuperado

de http://www.comie.org.mx/revista/v2018/rmie/index.php/nrmie/article/view/25/25

Salinas, M. L., Posada, D. M. e Isaza, L. (2002). A propósito del conflicto escolar. Revista Educación y Pedagogía, 14(34), 245-273. Recuperado de https://aprendeenlinea.udea.edu.co/revistas/index.php/revistaeyp/article/view/32511 0

Vázquez, J. J. y Valenzuela, G. A. (2013). Del análisis institucional a la etnografía institucionalista. Entrevista a Patrick Boumard. Revista Electrónica de Investigación Educativa, 15(1), 1-17. Recuperado de http://redie.uabc.mx/vol15no1/contenidovazquezval.html

Velázquez, L. (2011). Los estudiantes y la violencia. México: Eikon. 


\begin{tabular}{|c|c|}
\hline Rol de Contribución & Autor (es) \\
\hline Conceptualización & $\begin{array}{l}\text { Santos Noé Herrera Mijangos (Principal) Dayana Luna Reyes } \\
\text { (Apoyo) Jorge Gonzalo Escobar Torres (Apoyo) Janet Serrano } \\
\text { Díaz (Apoyo) }\end{array}$ \\
\hline Metodología & $\begin{array}{l}\text { Santos Noé Herrera Mijangos (Principal) Dayana Luna Reyes } \\
\text { (Apoyo) }\end{array}$ \\
\hline Software & No aplica \\
\hline Validación & $\begin{array}{l}\text { Santos Noé Herrera Mijangos (Principal) Dayana Luna Reyes } \\
\text { (Apoyo) }\end{array}$ \\
\hline Análisis Formal & $\begin{array}{l}\text { Santos Noé Herrera Mijangos (Principal) Dayana Luna Reyes } \\
\text { Apoyo) Jorge Gonzalo Escobar Torres (Apoyo) Janet Serrano } \\
\text { Díaz (Apoyo) }\end{array}$ \\
\hline Investigación & $\begin{array}{l}\text { Santos Noé Herrera Mijangos (Principal) Dayana Luna Reyes } \\
\text { (Apoyo) Jorge Gonzalo Escobar Torres (Apoyo) Janet Serrano } \\
\text { Díaz (Apoyo) }\end{array}$ \\
\hline Recursos & Jorge Gonzalo Escobar Torres \\
\hline Curación de datos & Dayana Luna Reyes \\
\hline $\begin{array}{l}\text { Escritura - Preparación del } \\
\text { borrador original }\end{array}$ & Santos Noé Herrera Mijangos \\
\hline $\begin{array}{l}\text { Escritura - Revisión y } \\
\text { edición }\end{array}$ & $\begin{array}{l}\text { Santos Noé Herrera Mijangos (Principal) Jorge Gonzalo } \\
\text { Escobar Torres (Apoyo) }\end{array}$ \\
\hline Visualización & Santos Noé Herrera Mijangos \\
\hline Supervisión & Santos Noé Herrera Mijangos \\
\hline $\begin{array}{l}\text { Administración de } \\
\text { Proyectos }\end{array}$ & $\begin{array}{l}\text { Santos Noé Herrera Mijangos (Principal) Janet Serrano Díaz } \\
\text { (Apoyo) }\end{array}$ \\
\hline Adquisición de fondos & $\begin{array}{l}\text { Santos Noé Herrera Mijangos Dayana Luna Reyes } \\
\text { Jorge Gonzalo Escobar Torres Janet Serrano Díaz } \\
\text { Contribución igual }\end{array}$ \\
\hline
\end{tabular}

